\title{
Laser-Shock Compression of Rhodamine 6G Dye in Ethanol Solution Studied by Time-Resolved Fluorescence Spectroscopy
}

\author{
ICHIYANAGI Kouhei, IGARASHI Yoshitaka, MATSUDA Akitaka, KONDO Ken-ichi \\ and NAKAMURA Kazutaka G. \\ Materials and Structures Laboratory, \\ Tokyo Institute of Technology, R3-10, 4259 Nagatsuta, Midori, Yokohama, Kanagawa 226-8503, Japan \\ (Recieced 4 February 2004 / Accepted 28 April 2004)
}

\begin{abstract}
A laser shock wave is generated by the irradiation of a 10-ns pulsed laser beam using a plasma confinement target assembly. Nanosecond time-resolved fluorescence spectroscopy is performed on rhodamine-6G dye in an ethanol solution. The fluorescence spectra show a red shift under shock compression. The observed data demonstrate that the fluorescence of rhodamine-6G dye is suitable for use as a pressure scale for laser shock compression at the examined pressure range.
\end{abstract}

\section{Keywords:}

shock, laser, fluorescence, nanosecond, rhodamine

\section{Introduction}

Shock wave science has been providing important data at high pressures to geophysics, astrophysics, and materials science. In conventional shock experiments, guns are used to launch a flyer for generating the shock wave. However, operating of the gas gun is difficult and dangerous. Recently, a method of laser shock compression has been developed using a pulsed laser. By focusing a pulsed laser beam onto materials, laser ablation occurs and the shock wave is generated as a counterforce of the laser ablation. Because pulsed lasers can irradiate a wide range of energy from nanojoules to kilojoules per pulse at time domains ranging from femtoseconds to nanoseconds, a shock wave can be generated by the irradiation of a pulsed laser beam onto materials. We can carry out time-resolved, pump-probe type shock experiments, e.g., Raman and fluorescence spectroscopies [1-3], for measuring transient changes in molecules under shock compression at a time resolution of the laser pulse width.

The use of optical spectroscopy for examination of the microscopic response of shocked materials has received increased attention in recent years [4]. Fluorescence from impurity atoms, molecules, or ions can provide important information regarding the local changes around the impurities. Shen and Gupta [5] studied the fluorescence of rhodamine6G (R6G) dye in an ethanol solution under high pressures using the conventional gun system and found that the peak shift of the fluorescence of the R6G is suitable for use as a pressure scale under $2 \mathrm{GPa}$, where a ruby scale is not practical because the peak shift of the fluorescence $\mathrm{R}$ lines of the ruby is small [6]. The R6G pressure scale is considered useful for laser shock compression, because the samples used in laser shock experiments are usually small. However, the applicability of this method to laser shock compression is not straight forward because the temporal and spatial distributions of the laser shock are dependent on those of the laser beams.

In this paper, we studied time-resolved fluorescence spectroscopy on R6G dye in an ethanol solution under laser shock compression employing a plasma confinement target assembly.

\section{Experimental}

A pump and probe technique was used for the timeresolved fluorescence spectroscopy. Figure 1 shows a schematic of the experimental setup. A shock wave was generated by focusing a laser beam on the aluminum targets, which contact to the sample, using plasma confinement geometry. The fundamental light $(1,064 \mathrm{~nm})$ of a nanosecond Q-switched Nd:YAG (yttrium aluminum garnet: Powerlite Pulse, Continuum) laser, whose pulse width is $10 \mathrm{~ns}$, was used for shock generation. The spatial and temporal distributions of the laser beam are Gaussian. The shock generating fundamental light was focused on the target through a multilens array coupled with a normal lens in order to improve the Gaussian laser profile to a flat top profile. The diameter of the focused-laser spot was $1.25 \mathrm{~mm}$. The probe beam was a second harmonic light $(532 \mathrm{~nm})$ from a picosecond Nd:YAG laser (EKSPLA) whose pulse duration 


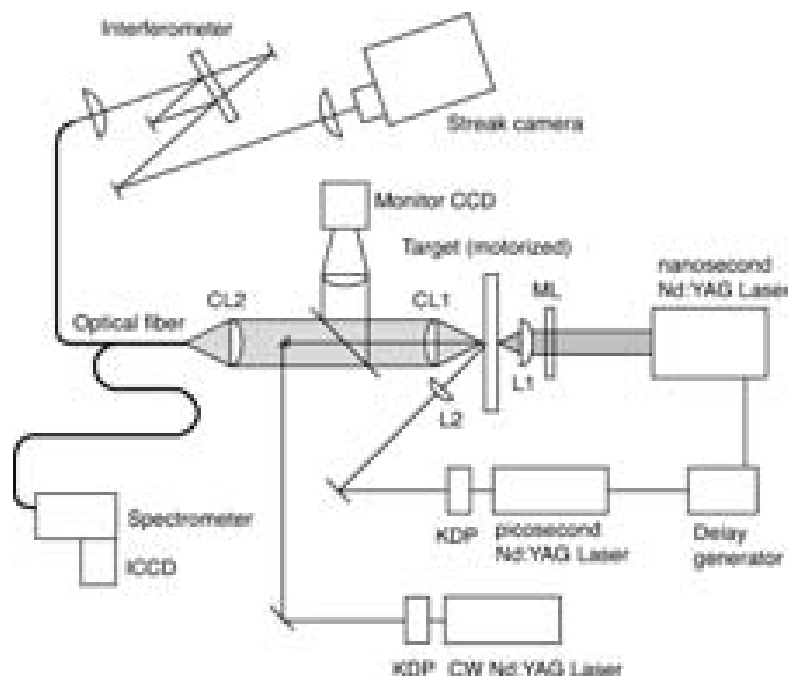

Fig. 1 Schematic of the experimental setup for velocity measurement. ML: multilens array, L1-2: lens, CL1-2: camera lens.

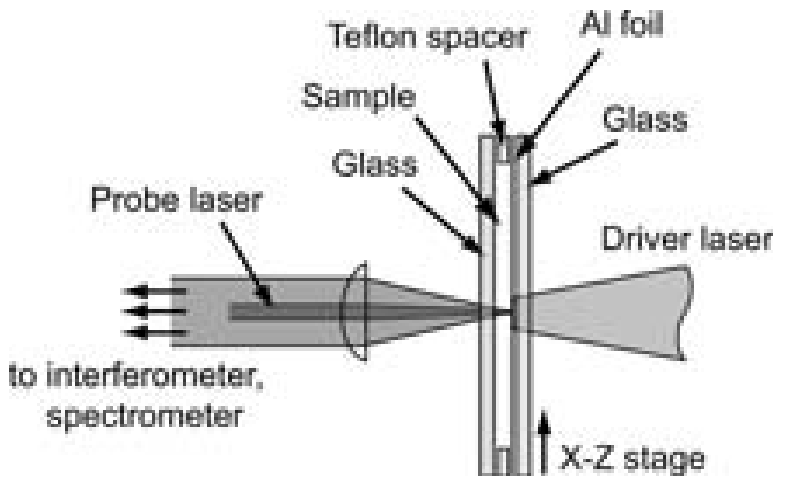

Fig. 2 Schematic of the target assembly.

is 25 ps. Both lasers were synchronized and delayed by using a pulse and delay generator (DG5353, Stanford Research). Timing jitter of the system is $\pm 1 \mathrm{~ns}$.

The target assembly with a plasma confinement geometry $[7,8]$ was fabricated with a back-up float glass substrate $\left(100 \times 100 \times 3 \mathrm{~mm}^{3}\right), 30-\mu \mathrm{m}$ thick aluminum foil, a $50-\mu \mathrm{m}$ thick Teflon spacer, and a cover float glass substrate $\left(100 \times 100 \times 3 \mathrm{~mm}^{3}\right)$. The sample was filled into the space surrounded by the Teflon and glasses (Fig. 2). The target assembly was mounted on a motorized X-Z stage. By focusing the laser beam onto the aluminum foil with a spot, confined plasma is generated near the aluminum-glass interface, which drives a shock wave through the aluminum foil into the sample. The sample was rohdamine-6G (R6G) in an ethanol solution at a concentration of $10^{-3} \mathrm{M}$. Both R6G and ethanol were spectroscopic grade. The probe laser beam was focused (a diameter of $200 \mu \mathrm{m}$ ) on the rear side of the sample and was centered opposite the focus of the pump beam. The energy of the probe pulse was set to be lower than $30 \mu \mathrm{J}$. Heating of the sample by the probe-laser pulse is

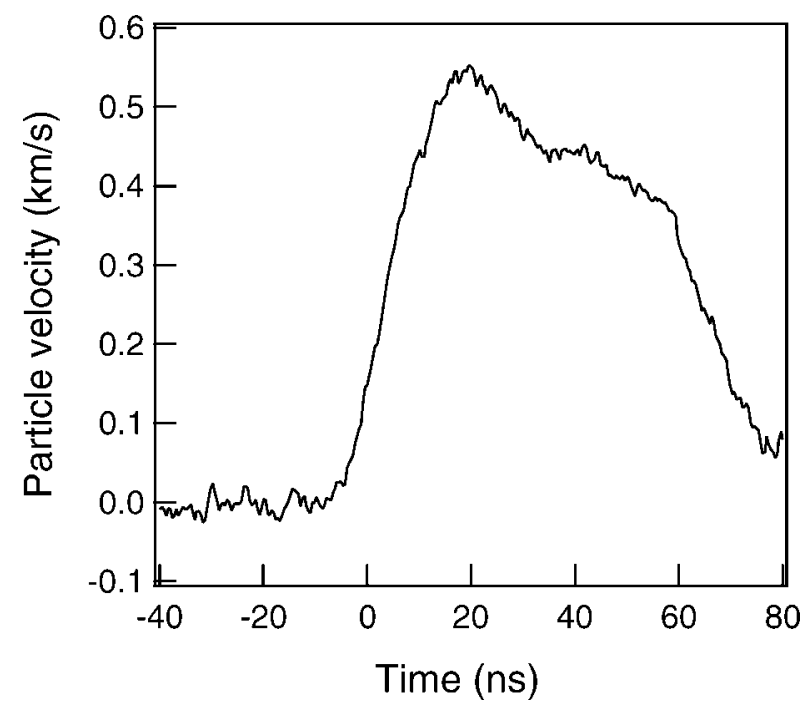

Fig. 3 The time evolution of the particle velocity at the interface between ethanol and the aluminum under laser irradiation at $500 \mathrm{~mJ}$.

negligible, because the laser energy is small and the ethanol is transparent for $532 \mathrm{~nm}$ light. The fluorescence was collected and focused into a $400 \mu \mathrm{m}$ core optical fiber by the camera lenses, introduced into a spectrometer, and detected with an intensified charge-coupled-device (CCD) camera (Andor Technology). The fluorescence signal is strong enough to be detected by a single shot experiment without any accumulation.

The particle velocity at the interface between the $\mathrm{Al}$ foil and the ethanol was independently measured using an optical recording velocity interferometer system (ORVIS) $[9,10]$. Using the particle velocity and an equation of state (EOS), we estimated the shock pressure. The EOS used was $U_{\mathrm{s}}=$ $1.37+1.65 u_{\mathrm{p}}$, which was calculated from data reported by Brown et al. [13], where $U_{\mathrm{s}}$ and $u_{\mathrm{p}}$ are the shock and particle velocities in $\mathrm{km} / \mathrm{s}$, respectively.

\section{Results and Discussions}

Figure 3 shows a typical example of the particle velocity obtained by the ORVIS measurement at the interface between the ethanol and the Al foil at a pump-laser energy of $500 \mathrm{~mJ}$. The maximum particle velocity obtained was $0.53 \pm 0.08$ $\mathrm{km} / \mathrm{s}$ and the peak pressure was estimated to be $0.93 \pm 0.22$ $\mathrm{GPa}$. The shock wave is generated by laser ablation of the $\mathrm{Al}$ foil. The laser-induced plasma is considered to be confined in the gap between the front glass and the Al foil, and the shock pressure was maintained for a relatively long time. In the plasma confinement target, shock duration $(\sim 40 \mathrm{~ns}$ in the present experiment) is longer than the pulse width of the pump laser beam (10 ns).

Figure 4 shows a typical example of the fluorescence spectra of R6G under shock compression at a peak pressure of $0.93 \pm 0.22 \mathrm{GPa}$. At the ambient pressure, the fluorescence has a peak at $559 \mathrm{~nm}$ and is assigned to the $\pi^{*}-\pi$ transition 


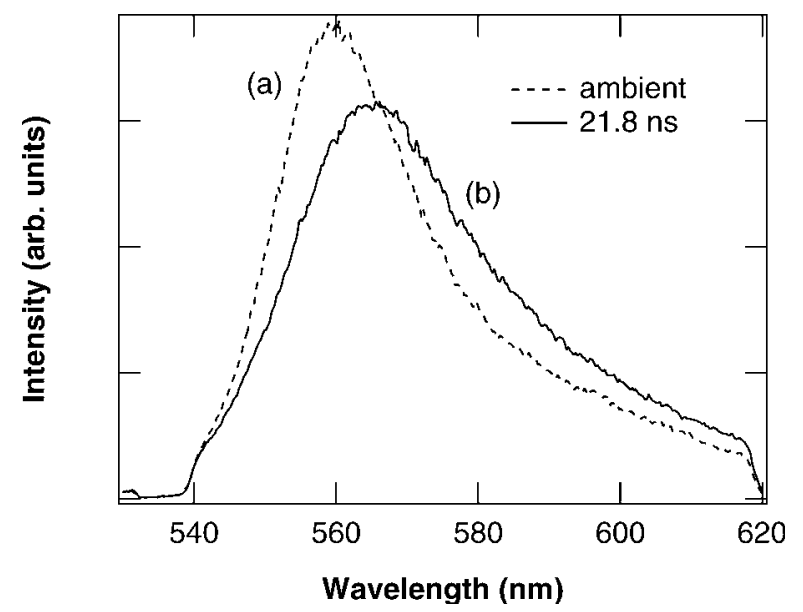

Fig. 4 Fluorescence spectra of rhodamine-6G in ethanol solution at ambient pressure (a) and at shock pressure of $0.93 \pm 0.22 \mathrm{GPa}(\mathrm{b})$.

[11]. At $0.93 \mathrm{GPa}$, the peak shows a red shift of $8 \mathrm{~nm}(210$ $\mathrm{cm}^{-1}$ ), which means that the energy gap between $\pi^{*}$ and $\pi$ states decreases. This is due to an increase of the polarization of the R6G molecule caused by increasing the pressure. At a delay time of $21.8 \mathrm{~ns}$, almost the whole volume of the R6G/ ethanol solution is considered to be under high pressure states. The shift of the fluorescence agrees closely with that reported by Shen and Gupta through the conventional shock experiment [5]. In addition to the red shift of the peak, the band width became boarder under shock compression. The spectral broadening is due to the increases in both pressure and temperature, but the peak shift is due mainly to the increase in the shock pressure [5]. The calculated shock temperature of the liquid ethanol at $0.93 \mathrm{GPa}$ was approximately $350 \mathrm{~K}$.

In the present measurement, time-resolution is approximately $4 \mathrm{~ns}$, because the lifetime of R6G in ethanol solution is $3.5 \mathrm{~ns}$ [12]. The spectra shown in Fig. 4 consist of fluorescence from both the shocked and ambient regions. The volume of the shocked region increases as the delay time increases, because the shock wave propagates inside the solution. Therefore, the ratio $\left(F_{\mathrm{s}} / F_{\mathrm{a}}\right)$ between the fluorescence from the shocked and the ambient regions changes. This ratio is expected to correspond to the volume ratio of the shocked and ambient regions and is expressed by

$$
\frac{F_{\mathrm{s}}}{F_{\mathrm{a}}}=\frac{V_{\mathrm{s}}}{V_{\mathrm{a}}}=\frac{U_{\mathrm{s}} t}{\left(d-U_{\mathrm{s}} t\right)},
$$

where $V_{\mathrm{s}}$ and $V_{\mathrm{a}}$ are volumes of the shocked and ambient region, respectively, $d$ is the thickness of the solution, and $U_{\mathrm{s}}$ is the shock velocity.

Figure 5 shows the time-resolved differential spectra of the R6G/ethanol solution at a peak shock pressure of $0.93 \pm$ $0.22 \mathrm{GPa}$. The intensity of the fluorescence signal increases as the delay time increases along with the shock wave propagation inside the solution. The fluorescence shifts to a

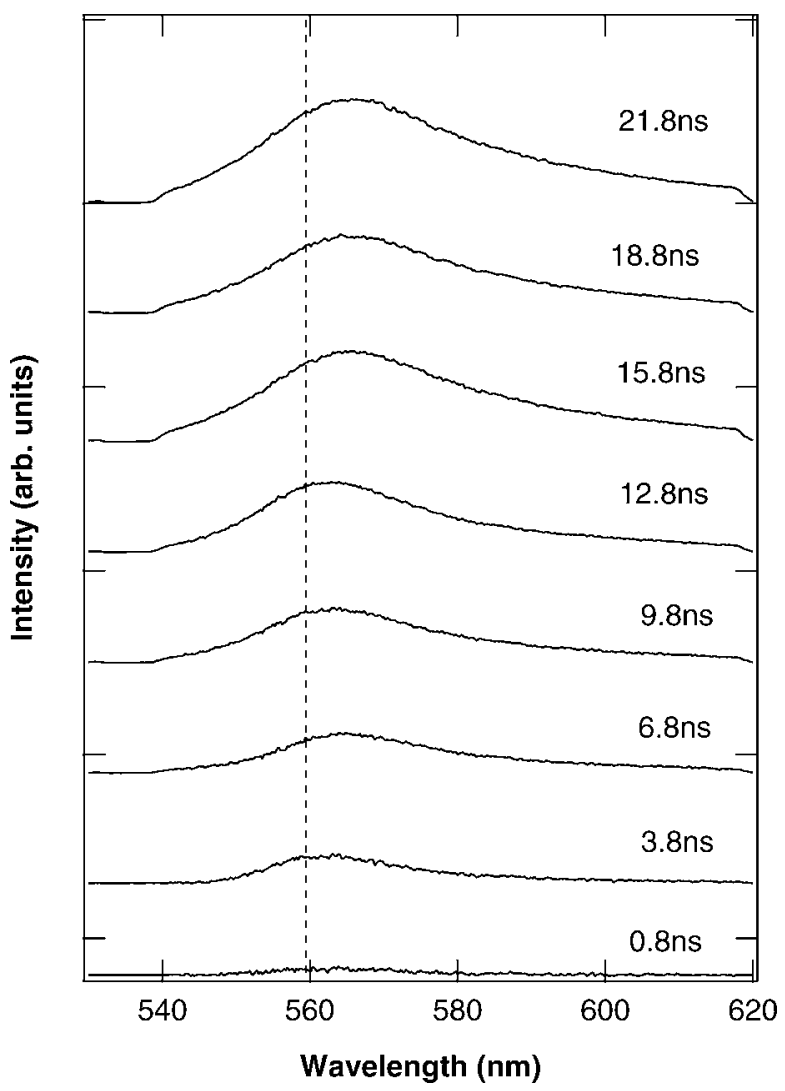

Fig. 5 Time-resolved differential fluorescence spectra of rhodamine- $6 \mathrm{G}$ in ethanol solution at shock pressure of $0.93 \pm 0.22 \mathrm{GPa}$.

higher wavelength of $8 \mathrm{~nm}\left(210 \mathrm{~cm}^{-1}\right)$ and broadens to 6.2 $\mathrm{nm}$ at full width of half maximum. The spectral shape does not change within the propagation of the shock wave for 20 ns. This means that shock compression is maintained for 20 $\mathrm{ns}$, which is longer than the pulse width (10 ns) of the laser beam, due to the effect of plasma confinement between the Al foil and the glass plate. The results show that undesirable phenomena such as reorientation and solidification of the solution do not occur at the present pressure. The timeresolved fluorescence spectroscopy measurements were also performed at higher-shock pressures of up to $1.6 \mathrm{GPa}$. At a shock pressure of $1.58 \pm 0.35 \mathrm{GPa}$, the spectral shape was almost similar to that at $0.93 \mathrm{GPa}$ and the peak shift was 17.2 $\mathrm{nm}\left(472 \mathrm{~cm}^{-1}\right)$. The obtained data suggest that the fluorescence shift is useful as a pressure scale for laser shock experiments at low pressure ranges. The relationship between the shock pressure $(P[\mathrm{GPa}])$ and the fluorescence peak shift $\left(F\left[\mathrm{~cm}^{-1}\right]\right)$ obtained was $F / P=-286 \pm 21$.

\section{Acknowledgment}

The authors thank M. Hasegawa for his help in constructing the experimental setup.

\section{References}

[1] H. Nagao, A. Matsuda, K.G. Nakamura and K. Kondo, Appl. Phys. Lett. 83, 249 (2003). 
[2] A. Matsuda, K.G. Nakamura and K. Kondo, Phys. Rev. B 65, 174116 (2002).

[3] A. Matsuda, H. Nagao, K.G. Nakamura and K. Kondo, Chem. Phys. Lett. 372, 911 (2003).

[4] D.E. Hare and D.D. Dlott, Appl. Phys. Lett. 64, 751 (1994).

[5] X.A. Shen and Y.M. Gupta, J. Appl. Phys. 70, 7552 (1991).

[6] G.J. Piermarini, S. Block, J.D. Barnett and R.A. Forman, J. Appl. Phys. 46, 2774 (1975).

[7] D. Devaux, R. Fabbro, L. Tollier and E. Bartnicki, J. Appl. Phys. 74, 2268 (1993).

[8] A. Matsuda, T. Hongo, H. Nagao, Y. Igarashi, K.G.
Nakamura and K. Kondo, Sci. Tech. Adv. Mater. (in press).

[9] L.M. Barker and K.W. Schuler, J. Appl. Phys. 45, 3692 (1974).

[10] D.D. Bloomquist and S.A. Sheffield, J. Appl. Phys. 54, 1717 (1983).

[11] B. Zhang, M. Chandrasekhar and H.R. Chandrasekhar, Appl. Opt. 24, 2779 (1985).

[12] S. Kinoshita and N. Nishi, J. Chem. Phys. 89, 6612 (1988).

[13] J.M. Brown, L.J. Slutsky, K.A. Nelson and L.T. Cheng, Science 241, 65 (1988). 